\title{
Características asociadas al abuso sexual infantil en un programa de intervención especializada en Santiago de Chile
}

\section{Characteristics associated to child sexual abuse in a specialized intervention program from Santiago, Chile}

\author{
Esteban Gómez ${ }^{1}$, Bernardita Cifuentes y Catalina Sieverson \\ Protectora de la Infancia, Santiago, Chile
}

(Recepción: Abril 2010 - Aceptación: Julio 2010)

\begin{abstract}
Resumen
Se presenta un estudio descriptivo de caracterización de los niños y niñas atendidos entre 2006 y 2009 en un programa de reparación de maltrato grave y abuso sexual infantil implementado por la Protectora de la Infancia y financiado por el Servicio Nacional de Menores de Chile. Se realizó un análisis retrospectivo de 100 fichas clínicas seleccionadas al azar (76 mujeres y 24 hombres), todos menores de 18 años. Los resultados muestran que se trata principalmente de niñas entre 8 y 12 años de edad, pertenecientes a familias de nivel socioeconómico bajo, con adecuados niveles de desarrollo salvo en la esfera socioemocional y psicosexual. El principal motivo de ingreso es abuso sexual, intrafamiliar, crónico, perpetrado en el propio hogar y por una figura masculina cercana a la niña(o) en la mayoría de los casos. La complejidad de estas situaciones demanda un enfoque integral y ecosistémico en la intervención.

Palabras claves: abuso sexual infantil, supuesto ofensor, programa de reparación, protección infantil.
\end{abstract}

\begin{abstract}
A descriptive study is presented, about the characteristics of boys and girls served between 2006 and 2009 in a child maltreatment and sexual abuse intervention program, implemented by Protectora de la Infancia and financed by the National Service of Children of Chile. A retrospective analysis was conducted on 100 clinical records randomly selected (76 women and $24 \mathrm{men}$ ), all of them less than 18 years old. The results show that they are mostly girls between 8 and 12 years old, from low income families, and with appropriate levels of development except on the socio-emotional and psycho-sexual sphere. The main intake motive is sexual abuse, intra-familial, chronic, perpetrated on their own home, and by a masculine figure close to the child, on most cases. The complexity of this situations demand an integral eco-systemic approach to the intervention.
\end{abstract}

Key words: child sexual abuse, supposed offender, intervention program, child protection.

1 Correspondencia a: Esteban Gómez. Avenida Concha y Toro, 1898, Puente Alto, Santiago de Chile. Teléfono: 4848975. E-mail: egomez@protectora.cl. 


\section{Introducción}

El maltrato infantil y el abuso sexual que experimentan niños, niñas y jóvenes cada año es un problema que no debe dejar a nadie indiferente. En Chile, se ha experimentado en la última década un proceso cultural paulatino de des-normalización de prácticas abusivas históricas, generándose una conciencia cada vez mayor respecto a los daños que dejan estas experiencias traumáticas y que repercuten a lo largo del desarrollo humano de no darse condiciones y procesos que promuevan la superación e integración del evento y sus consecuencias.

Los programas de reparación licitados por el Servicio Nacional de Menores [SENAME] en Chile a partir de 2004 son un reflejo de este proceso que aunque insuficiente, ha permitido abrir en los últimos años un tema mayoritariamente silenciado. La labor realizada por estos programas ha generado conocimiento relevante, tanto de la etiología y factores asociados al abuso, como de metodologías efectivas y pertinentes. Sin embargo, se identifica una escasez de publicaciones en Chile y Latinoamérica; en esta línea, Redondo y Ortiz (2005) reportaron sólo 41 artículos sobre Abuso Sexual Infantil (ASI) escritos en español, sobre un total de 8.043 disponibles en MEDLINE al año 2004, equivaliendo al $0,5 \%$ de la literatura publicada. Esto claramente impide transferir dicho conocimiento y ampliarlo a nuevos horizontes de práctica profesional en los programas de nuestros países.

Este estudio tuvo como objetivo identificar la problemática y el perfil del sujeto atendido en un programa especializado de Santiago de Chile en los últimos cuatro años de operatividad (20062009). Así, se caracterizó por una parte al niño víctima de maltrato y/o abuso sexual, la familia de la cual provenía, el supuesto agresor identificado y las principales características y consecuencias de la situación abusiva, según el juicio experto de los propios profesionales.

\section{Antecedentes}

Se estima que menos de un $20 \%$ de las víctimas de abuso sexual infantil denuncia el episodio por miedo a represalias, vergüenza, desconfianza en el servicio judicial, entre otras razones (Poblete \& Varas, 1994). A pesar de los problemas metodológicos en la evaluación del ASI (Almonte, Insunza \& Ruiz, 2002; Del Campo, 2003; Finkelhor, 1993; Pool, 2006), existen estudios de prevalencia a nivel internacional, siendo el más destacado el realizado por Finkelhor (1994), que comparó 21 investigaciones de diversos países principalmente norteamericanos y europeos, publicadas entre 1970 y 1990 , encontrando tasas entre un 7\% y un 36\% de ASI en mujeres y tasas entre un $3 \%$ y un $29 \%$ en hombres. Este estudio fue replicado, actualizado y ampliado por Pereda, Guilera, Forns \& GómezBenito (2009), encontrando entre $0 \%$ y $53 \%$ para mujeres y $0 \%$ y $60 \%$ para hombres.

Si bien Chile no cuenta con estudios epidemiológicos de ASI, existen ciertas cifras orientadoras; así, un estudio del Ministerio del Interior de Chile (2008), señala que el 7\% de los niños(as) entre 11 y 17 años ha sufrido alguna forma de abuso sexual en su vida. Además, el Anuario Estadístico de SENAME (30 de Septiembre de 2009) registra un total de 6.157 abusos sexuales que representan un $7,9 \%$ de los niños, niñas y adolescentes vigentes en la red SENAME.

\section{Características asociadas al ASI}

Respecto a la edad en que se observa la mayor proporción de abusos sexuales, la literatura muestra una concentración entre los 6 y los 13 años de edad, siendo el grupo más vulnerable los preadolescentes entre 8 y 12 años (Finkelhor, 2005; Onostre, 2000; Urrego, 2007; Roesler \& WeissmannWind, 1994; López et al., 1994; Sánchez \& Martín, 2007). Las publicaciones internacionales reiteran en forma categórica que las mujeres reportan en mayor proporción haber sufrido abuso sexual que los hombres, con entre un 55 y 95\% de los casos (Pereda et al., 2009; Onostre, 2000; Páez \& Hernández, 2004; Sánchez \& Martín, 2007; Almonte, Insunza \& Ruiz, 2002; SENAME, 2009; ACHNU, 2006). 
De acuerdo a González et al. (2004), entre 90 a 95\% de los abusos sexuales son realizados por hombres y la edad promedio de los abusadores es de 26 años; siendo los adolescentes responsables de aproximadamente $20 \%$ de las violaciones y $30 \%$ a $50 \%$ de los casos de abuso sexual infantil. El ASI es intrafamiliar entre un $65 \%$ y un $85 \%$ en la literatura (Elliott, Browne \& Kilcoyne, 1995; Fernández et al., 2001; Almonte et al., 2002). La mayor proporción de agresores corresponde al padre biológico con cerca de $25 \%$ de los casos (Fernández et al., 2001; Almonte et al., 2002). Respecto al contexto de ocurrencia, la mayoría (97\%) se trata de un lugar habitualmente conocido y frecuentado por el pequeño (Almonte et al, 2002). Entre los lugares más frecuentes, se reporta la residencia de la víctima con $28 \%$ a $43 \%$ y la residencia del abusador entre $21 \%$ a $34 \%$; otros lugares son minoritarios (Almonte et al., 2002; ACHNU, 2006; Fernández et al., 2001; Sánchez \& Martín, 2007).

\section{El acto de violencia sexual}

En un estudio que describe las características de la población infantil de un centro de atención a víctimas de abusos sexuales en Barcelona (Pereda, Polo, Grau, Navales \& Martínez, 2007), el tipo de abuso más frecuente son los tocamientos con un $76,2 \%$; respecto a la frecuencia del abuso, éste se produce en más de una ocasión en un 94,7\%. En otro estudio realizado en un dispositivo especializado en ASI de Andalucía, se obtuvo que la conducta abusiva más repetida fueron los tocamientos en los genitales del niño/a en un $45,7 \%$ de los casos, en un $26,9 \%$ obligar a la víctima a realizarle tocamientos al ofensor y en casi un $20 \%$ sexo oral (Sánchez \& Martín, 2007).

\section{La develación o detección del ASI}

Respecto de la develación de la situación de abuso, se ha reportado que sólo un tercio de los sujetos develó el abuso antes de los 18 años y el promedio de edad de develación fue a los 25,9 años (Terry \& Tallon, 2004). En los niños(as) que sí develan, la principal vía de develación del ASI es la familia, fundamentalmente la madre. Estudios realizados en España (Del Campo, 2003; Sánchez \& Martín, 2007; Pereda et al., 2007), han mostrado que la madre se constituyó en la figura de develación en el 34\% - 50,9\% de los casos, seguido por las fuentes profesionales, con 31,8\% - 57\%. La causa de descubrimiento suele ser el propio relato del niño con $50 \%$ de los casos; mientras que el descubrimiento por indicios físicos fue en $17 \%$, por indicios de anomalías en el comportamiento del niño en $14 \%$, por indicios sociales en $11 \%$, por la existencia de un ambiente de abuso en $6 \%$ y el resto de los casos (8\%) por descubrimiento en el mismo acto (Del Campo, 2003).

\section{Sintomatología asociada al ASI}

Dependiendo de la cronicidad, severidad y significación de la situación abusiva, la sintomatología variará (Huerta, Maric \& Navarro, 2002). En la etapa de inicio, predomina el Trastorno por Estrés Agudo y el Trastorno por Estrés Post Traumático (TEPT), mientras que en la fase crónica se ha señalado que prevalece el síndrome de acomodación a la victimización reiterada (Intebi, 1998). Los problemas más comúnmente observados son depresión o ansiedad, que se dan en un $29 \%$ de los casos; luego, comportamiento sexual inapropiado en un $17 \%$, problemas comportamentales en un $14 \%$, grupo de pares que genera mala influencia en un $13 \%$ y asistencia irregular al colegio en un 10\% (Trocmé et al., 2001). En nuestro país, Navarro, Carrasco y Aliste (2003) encontraron en niños y niñas chilenos con ASI la presencia (no sumativa) de $85 \%$ de síntomas ansiosos, $57 \%$ de síntomas depresivos, $49 \%$ de trastornos del sueño, $49 \%$ de defectos en la atención/concentración, $43 \%$ de conductas evitativas, $36 \%$ de auto/heteroagresividad, $28 \%$ de defectos en el control de la impulsividad, $26 \%$ de alteración de la conducta sexual, $23 \%$ de distorsiones cognitivas y un $23 \%$ de alteraciones de la conducta alimentaria.

\section{El programa CAM}

El Centro de Atención al Niño Maltratado (CAM) "Santa María Goretti" de la Protectora de la Infancia opera desde Mayo del 2004, habiendo atendido hasta el 31 de diciembre de 2009 un total 
de 359 casos. El objetivo de este programa es reparar integralmente las secuelas del maltrato y/o abuso sexual infantil; para ello, realiza acciones clínicas, psicosociales y sociolegales, en tres líneas metodológicas interconectadas desde un enfoque ecosistémico: la protección integral del niño, niña o joven gravemente vulnerado, la resignificación del daño y desculpabilización en espacios terapéuticos especializados y la restitución de derechos en su entorno familiar y social, propiciando una cultura de buen trato, cuidado y vinculación basada en el cariño y límites saludables.

\section{Método}

Se realizó un análisis retrospectivo de 100 fichas clínicas de casos atendidos en un programa de atención especializada en maltrato infantil grave y abuso sexual de la Protectora de la Infancia ubicado en la comuna de Puente Alto, Santiago de Chile. El período considerado abarcó entre enero de 2006 y diciembre de 2009, y la muestra representó el 43,3\% del total efectivamente atendido en ese período. Los casos fueron seleccionados al azar, con un total de 76 mujeres y 24 hombres, todos menores de 18 años.

El estudio replicó la mayor parte de la tipología usada por Sánchez y Martín (2007) para la consigna de las variables. A cada ficha se le asignó un folio o número de identificación, de manera de resguardar la confidencialidad de la información contenida en ellas. Se consideró cuatro instrumentos de las fichas: Informe de Calificación de la Agresión, Informe Social de Profundización Diagnóstica, Informe Psicológico de Profundización Diagnóstica y los Informes Periciales de las organizaciones derivantes. Estos instrumentos fueron diseñados y utilizados por los operadores del programa durante el período que abarca el estudio, y su información representa el juicio experto de dichos profesionales y/o de las organizaciones que derivan los casos (OPD, Tribunales de Familia, URAVIT, entre otras). Para el análisis de la información se utilizaron técnicas de estadística descriptiva básica.

\section{Resultados}

La caracterización de los niños(as) atendidos entre 2006 y 2009, muestra una edad promedio de 10,2 años $(\mathrm{SD}=3.87)$ con un mínimo de 1 y un máximo de 18 años; el $20 \%$ tiene 7 años o menos, el $40 \%$ tiene 8,6 años o menos, el $60 \%$ tiene 11 años o menos y el $80 \%$ tiene 14 años o menos. Se registra un $76 \%$ de sexo femenino y $24 \%$ de sexo masculino. Respecto a su escolaridad se observa que un 2,2\% asistía a jardín infantil o sala cuna, un 9,0\% a educación preescolar (pre-kinder o kínder), un mayoritario $32,6 \%$ se encontraban entre $1^{\circ}$ y $3^{\circ}$ básico, un $19,1 \%$ entre $4^{\circ}$ y $6^{\circ}$ básico, un $12,4 \%$ entre $7^{\circ}$ y $8^{\circ}$ básico y un $23,6 \%$ cursaban entre $1^{\circ}$ y $4^{\circ}$ medio, mientras que solo un $1,1 \%$ no se encontraba inscrito en ningún establecimiento. La asistencia se consigna como regular para el $87,1 \%$ de los casos.

La composición de las familias registra un promedio de 2,28 adultos $(\mathrm{SD}=1.19)$ y 2,29 niños por familia ( $\mathrm{SD}=1.08$ ), con un rango entre 1-6 adultos y 1-5 niños por familia. Se trata mayoritariamente de estructuras familiares biparentales $(51,8 \%)$, seguidas por uniparentales $(22,4 \%)$, extensa $(8,2 \%)$, hogares de protección $(9,4 \%)$, familias de acogida $(7,1 \%)$ y una figura significativa $(1,2 \%)$. Las familias registran un ingreso familiar $(\mathrm{N}=64)$ promedio de $\$ 300.015(\mathrm{SD}=241.797)$, perteneciendo en su mayoría a los grupos socioeconómicos más desfavorecidos de la población.

$\mathrm{Al}$ analizar su dinámica, se observa los límites familiares difusos en un $68,5 \%$, claros en un $27,8 \%$ y rígidos en un 3,7\%. Las jerarquías familiares se observan funcionales en un 39,6\% y disfuncionales en un $60,4 \%$. Las fronteras de la familia con su entorno se identifican como permeables en $40,0 \%$ de los casos, adecuadas en un $22,0 \%$ e impermeables en un $38,0 \%$. Respecto a los roles al interior de la familia se valoran como rígidos en un $53,8 \%$, inadecuados en un $28,8 \%$ y flexibles para el $15,4 \%$ de los casos atendidos. Cabe señalar que se observan problemas de violencia intrafamiliar en un $46,7 \%$ de los casos, y un $32,8 \%$ de los integrantes de la familia tienen algún problema de salud reportado. 


\section{Organizaciones que derivan los casos al programa CAM}

El análisis de las instituciones que derivaron los casos entre 2006 y 2009 se muestra en la Tabla 1. Destacan como principales derivantes las Oficinas de Protección de Derechos a la Infancia (OPD) con $29,3 \%$, seguido por las redes del sistema judicial que suman $42,4 \%$ (distribuidas en $19,6 \%$ de la URAVIT, 13\% de Tribunales de Familia y 9,8\% de las Fiscalías).

Tabla 1: institución que deriva al CAM (2006-2009)

\begin{tabular}{lcc}
\hline Institución que deriva el caso & Frecuencia & Porcentaje \\
\hline Oficina de Protección de Derechos & 27 & $29,3 \%$ \\
URAVIT & 18 & $19,6 \%$ \\
Tribunal de Familia & 12 & $13,0 \%$ \\
Red SENAME & 10 & $10,9 \%$ \\
Fiscalía & 9 & $9,8 \%$ \\
Red Interna & 7 & $7,6 \%$ \\
Otros & 9 & $9,8 \%$ \\
\hline Total & 92 & $100,0 \%$ \\
\hline
\end{tabular}

En menor proporción aparece la red de programas del Sename con 10,9\% y la propia red interna de derivación con 7,6\%. Otras redes como el COSAM y otras, conforman un 10\% de las derivaciones. Para 8 casos no se dispuso de esta información. Es importante clarificar que todos los casos ingresaron al programa sin una condena emitida por Tribunales, lo que implica que se trata de casos con sospecha y denuncia por abuso, no de casos confirmados judicialmente. Sin embargo, todos los casos incorporados en el estudio tienen antecedentes psicosociales contundentes para asumir la veracidad de la denuncia y trabajar sobre esta base.

Tabla 2: tipo de agresión, por sexo del niño(a) y del agresor

\begin{tabular}{|c|c|c|c|c|c|c|}
\hline & & \multicolumn{5}{|c|}{ Tipo de Agresión } \\
\hline & & $\begin{array}{l}\text { Abuso } \\
\text { Sexual }\end{array}$ & Violación & $\begin{array}{c}\text { Maltrato } \\
\text { Físico }\end{array}$ & $\begin{array}{c}\text { Maltrato } \\
\text { Emocional }\end{array}$ & Negligencia \\
\hline \multirow{2}{*}{\multicolumn{2}{|c|}{$\begin{array}{l}\text { TOTAL Niños \& Niñas } \\
(\mathrm{N}=100)\end{array}$}} & 82 & 30 & 23 & 13 & 8 \\
\hline & & $82 \%$ & $30 \%$ & $23 \%$ & $13 \%$ & $8 \%$ \\
\hline \multirow{6}{*}{$\begin{array}{l}\text { Sexo } \\
\text { Niño }\end{array}$} & Mujer $(n=76)$ & 62 & 23 & 14 & 8 & 7 \\
\hline & $\%$ sobre $\mathrm{N}$ mujer & $81,6 \%$ & $30,3 \%$ & $18,4 \%$ & $10,5 \%$ & $9,2 \%$ \\
\hline & $\begin{array}{l}\text { \% sobre Tipo de } \\
\text { Agresión }\end{array}$ & $75,6 \%$ & $76,7 \%$ & $60,9 \%$ & $61,5 \%$ & $87,5 \%$ \\
\hline & Hombre $(\mathrm{n}=24)$ & 20 & 7 & 9 & 5 & 1 \\
\hline & $\begin{array}{l}\% \text { sobre } \mathrm{N} \\
\text { hombre }\end{array}$ & $83,3 \%$ & $29,2 \%$ & $37,5 \%$ & $20,8 \%$ & $4,2 \%$ \\
\hline & $\begin{array}{l}\text { \% sobre Tipo de } \\
\text { Agresión }\end{array}$ & $24,4 \%$ & $23,3 \%$ & $39,1 \%$ & $38,5 \%$ & $12,5 \%$ \\
\hline
\end{tabular}




\begin{tabular}{|c|c|c|c|c|c|c|}
\hline \multicolumn{7}{|l|}{ Sexo } \\
\hline \multirow[t]{10}{*}{ Agresor } & Mujer $(n=9)$ & 4 & 1 & 7 & 6 & 5 \\
\hline & $\begin{array}{l}\% \text { sobre } \mathrm{N} \text { mujer } \\
\% \text { sobre Tipo de }\end{array}$ & $44,4 \%$ & $11,1 \%$ & $77,8 \%$ & $66,7 \%$ & $55,6 \%$ \\
\hline & Agresión & $4,9 \%$ & $3,3 \%$ & $30,4 \%$ & $46,2 \%$ & $62,5 \%$ \\
\hline & Hombre $(\mathrm{n}=88)$ & 75 & 27 & 16 & 6 & 3 \\
\hline & $\%$ sobre $\mathrm{N}$ & & & & & \\
\hline & hombre & $85,2 \%$ & $30,7 \%$ & $18,2 \%$ & $6,8 \%$ & $3,4 \%$ \\
\hline & $\begin{array}{l}\text { \% sobre Tipo de } \\
\text { Agresión }\end{array}$ & $91,5 \%$ & $90,0 \%$ & $69,6 \%$ & $46,2 \%$ & $37.5 \%$ \\
\hline & Datos perdidos & & & & & \\
\hline & $\%$ sobre Tipo de & 3 & 2 & 0 & 1 & 0 \\
\hline & Agresión & $3,6 \%$ & $6,7 \%$ & $0 \%$ & $7,6 \%$ & $0 \%$ \\
\hline
\end{tabular}

Fuente: elaboración propia de los autores.

\section{Tipo de agresión y frecuencia}

El tipo de maltrato identificado al ingreso, se distribuyó en un $82 \%$ de abuso sexual, seguido por un $30 \%$ de violación, $23 \%$ de maltrato físico, $13 \%$ de maltrato emocional y $8 \%$ de negligencia, no registrándose casos atendidos por abandono o explotación. La Tabla 2 cruza el sexo del niño y el tipo de agresión, mostrando la proporción respecto al total de cada tipo de agresión, así como la distribución de los tipos agresión al interior de cada sexo.

Es importante clarificar que estas agresiones pueden combinarse entre sí, ya que los niños(as) atendidos registran entre 1 y 4 tipos de agresiones, promediando $1,56(\mathrm{SD}=0.86)$; un $64 \%$ recibió un único tipo de maltrato, $20 \%$ dos tipos de maltratos, $12 \%$ recibió tres tipos de maltrato, y solamente un $4 \%$ registró cuatro tipos de maltrato diferentes.

\section{Características del agresor/a}

La edad del supuesto agresor promedia 28,6 años $(\mathrm{SD}=17.94)$, con un mínimo de 10 y un máximo de 72 años. Se registra un $35,8 \%$ de supuestos agresores menores de 17 años $(19,4 \%$ entre 10 y 14 años y $16,4 \%$ entre $14-17$ años), un $53,7 \%$ entre 18 y 55 años y un $10,4 \%$ mayores de 55 años. El sexo del supuesto agresor es mayoritariamente masculino con 90,7\%; la Tabla 2 muestra la relación entre tipos de agresión y sexo del agresor, observándose que la proporción de ofensores hombres que agreden sexualmente a los niños es muy superior a la proporción entre las ofensoras, mientras que la proporción de maltrato físico/emocional y negligencia es superior entre las ofensoras mujeres.

La identificación del supuesto agresor sexual en los casos atendidos, contabilizó un total de 119 agresores. Se observa que el principal agresor sería el padre con 18,5\%, seguido por el padrastro con $12,6 \%$ y la pareja de figuras femeninas significativas de la familia con 9,2\%. El resto de los supuestos ofensores se reparte en una amplia gama de personas cercanas al niño o niña agredido, como primos, hermanos, madres, figuras de la comunidad, vecinos, abuelos, tíos, niños de la escuela o desconocidos.

El vínculo del niño con el supuesto agresor es principalmente intrafamiliar consanguíneo $(51,5 \%)$, seguido por extrafamiliar conocido $(25,3 \%)$, intrafamiliar no consanguíneo $(19,2 \%)$, extrafamiliar desconocido con solamente un 1,0\% y se desconoce en 3,0\%. Al analizar el cruce entre el sexo del niño(a) y el vínculo con el agresor, se observa que en las niñas un $74 \%$ de las agresiones 
son intrafamiliares, mientras que un $24 \%$ son extrafamiliares. Esta distribución es un tanto diferente de la observada en niños, donde el $61 \%$ es intrafamiliar y el $35 \%$ son extrafamiliares.

\section{Características de la agresión}

Los malos tratos ejercidos sobre el niño(a) consistieron en eventos únicos para el $28,2 \%$ de los casos, mientras que un mayoritario $71,8 \%$ registra eventos reiterados. La agresión ejercida sobre el niño sería transgeneracional en $27 \%$ a $60 \%$ de los casos. El modo empleado por el supuesto abusador fue principalmente mediante amenazas en un $83 \%$, seguido por seducción en un $10 \%$ y ambas en un 7\%. Respecto al lugar de ocurrencia del abuso, principalmente se trata de la casa del propio niño(a) con $65,4 \%$, seguida por otros lugares distintos con $19,2 \%$ y la casa del ofensor distinta a la del niño con $15,4 \%$ de los casos.

El análisis de las principales acciones abusivas realizadas por los supuestos agresores en el período total cubierto por el estudio muestra que lo más frecuente fueron tocamientos en órganos sexuales por debajo de la ropa en el $64,6 \%$ de los casos, seguido por penetración con pene anal y/o vaginal con $35,4 \%$, tocamientos en órganos sexuales por encima de la ropa con $13,9 \%$, obligar a la víctima a realizar tocamientos en genitales del ofensor con $12,7 \%$, sexo oral con $11,4 \%$ y frotamientos genitales en $6,3 \%$ de los casos. No se registraron situaciones de exhibicionismo o voyerismo.

\section{Develación y culpa en el niño}

Los indicadores que alertaron a las organizaciones de la red respecto a una situación de agresión sexual en los casos derivados, muestran que en un 79\% de los niños se identificaron indicadores psicológicos inespecíficos como depresión/tristeza o cambios repentinos de conducta, un $61 \%$ exhibió indicadores conductuales/relacionales inespecíficos como dificultades en la escuela, solamente en un $31 \%$ se detectaron indicadores psicosexuales específicos como conducta o lenguaje erotizado y para un mayoritario $78 \%$ de los casos el principal indicador fue la verbalización por parte del niño de haber sido abusado, constituyendo el grupo de casos en que hubo develación, observándose en el restante $22 \%$ el descubrimiento de la situación por parte de un tercero.

La persona depositaria de la develación fue mayoritariamente la madre en un 54,4\% de los casos, seguida por otras personas significativas de su entorno más íntimo como la abuela(os) con $8,9 \%$, una amiga(s) o compañera de colegio con 7,6\%, una hermana(o) con 6,3\%. El resto de las situaciones registra frecuencias menores (padre, profesor del colegio, profesional de las ciencias sociales y otras figuras femeninas con un 5,1\% cada uno). En general, las personas a las que los niños(as) y adolescentes acudieron para develar la agresión vivida resultaron ser figuras femeninas.

Como elementos necesarios para el óptimo desarrollo del proceso de intervención del programa CAM, se observó la presencia de un adulto significativo en un 96,5\% de los casos, siendo principalmente intrafamiliar con $97,6 \%$. La reacción del adulto significativo frente a la develación se identificó como mayoritariamente positiva por cuanto le creyó al niño y se movilizó en un $83,1 \%$ de las situaciones, mientras que resultó negativa en un $16,9 \%$. Cabe destacar que la escuela conocía la situación de agresión en un $85,5 \%$ de las situaciones.

En los niños(as) y adolescentes atendidos se identificó conciencia de la situación abusiva en un significativo $91,7 \%$ de los casos, mientras que de acuerdo al juicio experto de los profesionales existía un sentimiento de culpa asociado a la situación de ASI en el $64 \%$ de los niños y niñas ingresados.

\section{Diagnóstico y Secuelas observadas en los niños(as) y adolescentes atendidos}

Como muestra el gráfico 1 , los informes psicodiagnósticos revelan que el nivel de pensamiento e intelectual estaría dentro de parámetros normales para la gran mayoría de los niños(as) y adolescentes atendidos; lo mismo se observa para el desarrollo del lenguaje, el desarrollo psicomotor y el desarrollo físico de los niños. Las dimensiones más alteradas son aquellas que se encuentran más 
directamente ligadas a la experiencia abusiva vivida por los niños(as) y adolescentes, con solamente un $50 \%$ de los casos dentro de parámetros normales de desarrollo psicosexual y $19 \%$ de desarrollo emocional.

Gáfico 1: Desarrollo Integral en los niños(as) atendidos por ASI

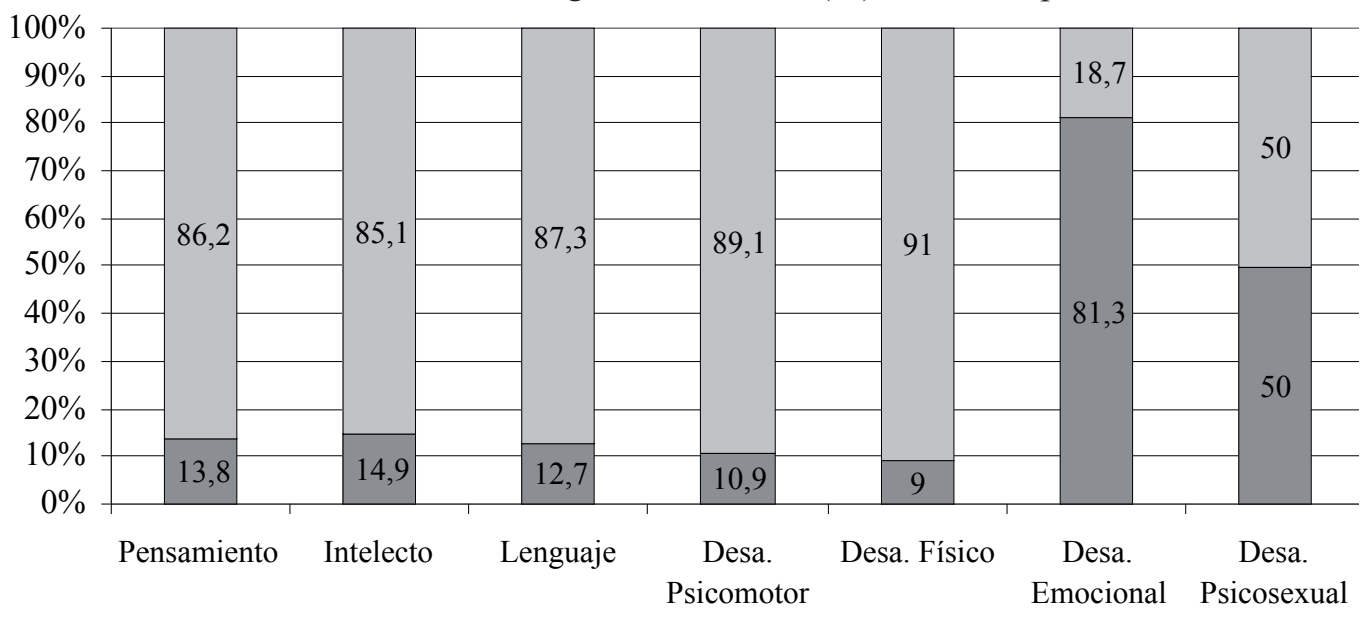

\section{Alterado $\square$ No Alterado}

De acuerdo a los diagnósticos realizados por los profesionales del CAM, los rasgos de personalidad de los niños serían depresivos (43\%) y evitativos (22\%) en su mayoría, con menor presencia de rasgos dependientes $(16 \%)$, limítrofes $(10 \%)$, histriónicos $(5 \%)$, esquizoides $(2 \%)$ y obsesivocompulsivos (2\%). Respecto a la capacidad de relación interpersonal de los niños(as) y jóvenes atendidos, se observan dificultades para establecer límites, tanto con sus pares (54\%) como con los adultos $(61 \%)$, mientras que su capacidad para confiar en los adultos del entorno se observa dañada en el $38 \%$ de los usuarios del programa.

Tabla 3: síntomas identificados en los niños(as) y adolescentes tras su ingreso al CAM $(\mathrm{N}=100)$

\begin{tabular}{ccccccc}
\hline & $\begin{array}{c}\text { Síntomas de } \\
\text { Estrés Post } \\
\text { Traumático }\end{array}$ & $\begin{array}{c}\text { Problemas de } \\
\text { Agresividad - } \\
\text { Impulsividad }\end{array}$ & $\begin{array}{c}\text { Problemas } \\
\text { Psicosexuales }\end{array}$ & $\begin{array}{c}\text { Problemas } \\
\text { Aprendizaje }\end{array}$ & $\begin{array}{c}\text { Problemas } \\
\text { Pensamiento }\end{array}$ & $\begin{array}{c}\text { Problemas } \\
\text { Imagen } \\
\text { Corporal }\end{array}$ \\
\hline 2-4 síntomas & $26 \%$ & $13 \%$ & $5 \%$ & $7 \%$ & $0 \%$ & $0 \%$ \\
1 síntoma & $27 \%$ & $17 \%$ & $18 \%$ & $22 \%$ & $13 \%$ & $11 \%$ \\
\hline TOTAL & $53 \%$ & $30 \%$ & $23 \%$ & $29 \%$ & $13 \%$ & $11 \%$ \\
\hline
\end{tabular}

Nota 1. Los síntomas de Estrés Post Traumático considerados fueron: re-experimentación ( $\mathrm{n}=20)$, conductas evitativas $(n=16)$, síntomas disociativos $(n=8)$, hiper-arousal $(n=6)$, y trastornos del sueño $(n=37)$.

Nota 2. Los síntomas considerados en Agresividad - Impulsividad fueron: auto/hetero agresividad ( $\mathrm{n}=26)$, oposicionismo $(n=15)$, conductas disociales $(n=5)$ y abuso de sustancias $(n=2)$.

Nota 3. Los síntomas de Problemas Psicosexuales fueron: alteración de la conducta o identidad sexual ( $\mathrm{n}=16)$ y enuresis/encopresis secundaria al abuso $(n=12)$.

Nota 4. Los síntomas considerados en Aprendizaje fueron: déficit de atención/concentración ( $\mathrm{n}=20)$, hiperactividad $(\mathrm{n}=10)$ y alteración del lenguaje aprendizaje/lectoescritura/cálculo/psicomotor $(\mathrm{n}=6)$. 
Nota 5. Los síntomas considerados en Pensamiento fueron: distorsiones cognitivas ( $\mathrm{n}=13)$ y alucinaciones/ delirios $(\mathrm{n}=0)$.

Nota 6. Los síntomas considerados en Imagen Corporal fueron: alteración de la conducta alimentaria $(\mathrm{n}=10)$ y síntomas somatomorfos $(\mathrm{n}=1)$.

Se registró un promedio de 2,24 síntomas clínicos por niño ( $\mathrm{SD}=1.88$ ), con un 20\% que registra un síntoma, $20 \%$ dos síntomas, $13 \%$ tres síntomas, $15 \%$ cuatro síntomas y $11 \%$ que registran entre cinco a ocho síntomas. Cabe señalar que en un $21 \%$ adicional no se identificó ningún síntoma en las fichas de ingreso (esto implica que si se consideran solo los casos con registros de síntomas, el promedio de síntomas por niño sube a casi 3,0). Los resultados reflejados en la Tabla 3 sugieren que al ingresar al programa un 53\% de los niños mostraban algún síntoma de estrés post traumático, 30\% algún indicador sintomático de agresividad/impulsividad, 29\% algún problema para el aprendizaje, $23 \%$ algún indicador de problemas psicosexuales, $13 \%$ problemas en su lógica de pensamiento y un $10 \%$ exhibieron alteración de la conducta alimentaria.

Según la clasificación de tipología sintomática general diagnosticada, un 36,5\% mostraba sintomatología de tipo ansioso, un 29,7\% sintomatología de tipo depresivo, un 23,0\% sintomatología de tipo ansioso/depresivo, un 4,1\% mostraba síntomas de tipo maníaco y solo un 1,4\% síntomas de tipo bipolar. Solo para un 5,4\% se registró explícitamente la ausencia de sintomatología identificable o sintomatología remitida.

\section{Discusión}

El estudio mostró datos concordantes con la literatura revisada, que señala como edades de mayor riesgo entre los 8 y 12 años de edad (Finkelhor, 2005; Ministerio del Interior, 2008; Onostre, 2000; Urrego, 2007; Roesler \& Weissmann-Wind, 1994; López et al., 1994; Sánchez \& Martín, 2007) y una alta prevalencia de maltrato sexual en niñas por sobre los varones (Almonte, Insunza \& Ruiz, 2002; Sánchez \& Martín, 2007; Pereda et al., 2009; Ministerio del Interior, 2008; SENAME, 2009), reforzando la tesis de que ser niña en edad escolar es un factor de riesgo importante para el ASI.

En cuanto al tipo de agresión identificado al ingreso, se observó una altísima presencia de abuso sexual, por sobre violación, maltrato físico/emocional y negligencia, observándose la prevalencia de un tipo único de agresión en dos de cada tres casos. El análisis de las principales acciones realizadas por los supuestos agresores muestra que lo más frecuente fueron vejaciones sexuales, seguido por penetración, mostrando un patrón similar al de los casos atendidos en el estudio de Sánchez y Martín (2007), salvo por la ausencia de situaciones de exhibicionismo y voyerismo. Ante esta evidencia que señala al abuso sexual como forma de agresión preponderante entre los usuarios del programa CAM, queda de manifiesto no sólo la necesidad de contar con profesionales expertos en ASI, sino de mantener un programa de capacitación y actualización para el personal que sea altamente especializado (Santana \& Farkas, 2007).

La mayoría de estas agresiones ocurren en el espacio íntimo del niño o niña; se trata de familiares, conocidos, personas que componen su red más estrecha de relaciones sociales, de ahí que el daño sea mayor y que su visibilización se vea dificultada (Barudy, 1998). El presente estudio mostró hallazgos coherentes con la literatura respecto a que la agresión se da en un lugar habitualmente conocido y frecuentado por el niño (Almonte et al., 2002; ACHNU, 2006; Fernández et al., 2001), con tasas aun más altas de ocurrencia del ASI en el propio domicilio del niño(a), para la presente muestra.

Es importante reflexionar respecto al rol de la escuela en el afrontamiento del ASI, ya que en este estudio casi la totalidad de los niños y niñas atendidos en el programa permanecieron insertos en el sistema educativo, cursando niveles correspondientes a su edad y con asistencia regular. Esto hace pensar que si las agresiones vividas se llevan a cabo fundamentalmente en contextos familiares, la escuela constituye para estos niños un espacio protector y de gratificación, el cual no abandonan. 
Asimismo, abre las puertas a considerar este espacio como una oportuna plataforma de prevención y detección precoz, dado que los alumnos de educación básica son la población de mayor riesgo para el abuso sexual (Finkelhor, 2005).

\section{Contexto Familiar}

$\mathrm{Al}$ analizar la dinámica familiar, es posible determinar características distintivas de disfuncionalidad, que se ajustan a los modelos explicativos desarrollados por los diversos autores consultados y que refieren alteraciones en la organización y dinámica familiar de las víctimas (Barudy, 1998; Gladstone et al., 2004; González et al., 2004; Trepper \& Barret, 1989), especialmente la distorsión de roles y la difusión de límites entre sus miembros, transformando este entorno en poco seguro y protector del niño y donde se darían situaciones transitorias de estrés que favorecen los actos abusivos contra los miembros más débiles (Trepper, Niedner, Mika \& Barrett, 1994; Finkelhor, 2005; Beltrán, 2007).

Asimismo, se pudo identificar un componente transgeneracional que opera en dos niveles: por un lado, se trasmite un mayor riesgo de sufrir eventos abusivos cuando las madres lo han sufrido y por otro la tendencia a repetir patrones relacionales dañinos en las nuevas conformaciones familiares (Maida et al., 2005). Este fenómeno ha sido descrito en la literatura especializada como un predictor significativo de la reiteración del abuso en los hijos, cuando no ha mediado un tratamiento especializado; por ejemplo Maida et al. (2005), en un estudio reciente desarrollado en el Hospital Luis Calvo Mackenna en Santiago de Chile, encontró que las madres de niños abusados sexualmente presentaban mayor cantidad de reportes de abuso sexual en su infancia (61,4\% versus $26,7 \%)$, y maltrato físico y psicológico (72,7\% versus 33,3\%), en comparación con el grupo de control, observándose diferencias significativas para todos los tipos de maltrato y abuso sufridos en la infancia. Estos hallazgos, plantean un desafío al modelo de intervención clínico individual utilizado por muchos programas chilenos de intervención en ASI, donde el foco ha estado puesto casi exclusivamente en la reparación individual del niño y su desculpabilización, por sobre un modelo familiar sistémico que genere cambios en la organización y dinámica familiar, abordando a todos sus miembros (Sinclair \& Martínez, 2006).

\section{Desafíos de la intervención}

En consideración al hallazgo de que el lugar de ocurrencia del abuso sexual es principalmente la casa del propio niño (65\%), se levanta la interrogante sobre las implicancias que ello tiene en el tratamiento y en cómo trabajar la significación del espacio físico (su pieza, su cama, sus juguetes) si el niño habitualmente sigue viviendo en el lugar del abuso. En este sentido, resulta necesario remirar el uso y sentido otorgado a las visitas domiciliarias, así como la pertinencia de efectuar intervenciones clínicas en el hábitat del niño, con el niño y sus adultos significativos, orientadas a reorganizar el espacio de acuerdo a las nuevas significaciones adquiridas en su proceso de reparación.

Si bien casi la totalidad de los niños y niñas atendidos tiene consciencia de la trasgresión experimentada, se observó un sentimiento de culpa asociada en dos de cada tres casos (Finkelhor \& Browne, 1985). En este sentido, el foco técnico del programa en la desculpabilización parece pertinente, especialmente en aquellos que tuvieron un afrontamiento pasivo de la agresión. Sin embargo, trabajar con el niño la construcción de límites personales, sin trabajarlo con la familia como sistema podría ser un efecto no deseado de responsabilización del niño en el modelo de tratamiento.

En línea con otras investigaciones, la sintomatología observada en los niños es principalmente de tipo ansiosa y/o depresiva, organizándose mayoritariamente a través de cuadros de estrés postraumático, es decir, prevalecen sentimientos de indefensión, desamparo, desconfianza y temor como consecuencia de la agresión experimentada. Desde el modelo traumatogénico del ASI, estos síntomas levantan el desafío para los profesionales de conectar permanentemente las acciones socio-legales de protección frente a la agresión, con las resignificaciones trabajadas en el espacio clínico y familiar para restablecer el sentimiento de seguridad básica en el niño (Finkelhor \& Browne, 1985). 
Las agresiones de tipo sexual son efectuadas principalmente por sujetos de sexo masculino, lo que resulta plenamente concordante con la literatura revisada. En el $40 \%$ se trata del padre, padrastro o figura parental masculina, lo que puede ser un elemento que dificulte realizar acciones de protección si dicha figura es el único proveedor económico de la familia. En contraparte, la develación se da en $78 \%$ de los niños, siendo principalmente la madre (54\% de los casos), la persona escogida para confidenciar la situación vivida, seguida de otras figuras femeninas significativas de su entorno inmediato, fenómeno igualmente descrito en la literatura (Del Campo, 2003; Sánchez \& Martín, 2007; Pereda et al., 2007; Sinclair \& Martínez, 2006). La alta frecuencia del padre o figura parental masculina como agresor, combinado con la figura femenina como depositaria de la develación y protección, abren la interrogante respecto a la polarización de estereotipos de género que puede producirse en los niños, generando otro desafío para estos programas respecto a la necesidad de incorporar un enfoque de género orientado a reparar la significación del ser hombre/ser mujer en la intervención y estimulando el desarrollo de nuevos estudios en el tema.

Aun resulta difícil en nuestro país la detección y tratamiento tempranos del abuso sexual infantil; se hace indispensable, por tanto, explorar nuevos sistemas locales de detección precoz -tarea recientemente abordada por SENAME (Fundación León Bloy, 2008)- y desarrollar estudios que evalúen la claridad de los modelos de intervención en ASI propuestos, la congruencia de los procesos y acciones efectivamente desarrollados por los equipos profesionales y la calidad y sostenibilidad de los resultados obtenidos en los programas especializados en Chile y Latinoamérica.

\section{Referencias}

Almonte, C., Insunza, C. \& Ruiz, C. (2002). Abuso Sexual en Niños y Adolescentes de Ambos Sexos. Revista Chilena de Neuro-psiquiatría, 40(1), 22-30.

Barudy, J. (1998). El dolor invisible de la infancia. Una lectura ecosistémica del maltrato infantil. Barcelona: Paidós.

Beltrán, C. (2007). Características y factores precipitantes asociados al abuso sexual. Revisión Sistemática Universidad Autónoma de Bucaramanga, Bucaramanga, Colombia. Vol. $10 \mathrm{~N}^{\circ} 1$ Abril de 2007.

Chile, Ministerio del Interior (2008). Encuesta nacional de victimización por violencia intrafamiliar $y$ delitos sexuales. Santiago de Chile.

Chile, Servicio Nacional de Menores (2009). Niños (as) y adolescentes vigentes en la red Sename que registran algún tipo de maltrato o abuso sexual. Boletín temático. Tercer trimestre 2009. Disponible en: http://www.sename.cl/

Del Campo, A. (2003). Detección del abuso sexual a menores: definición, prevalencia, indicadores y factores de riesgo. Revista Pediátrica Atención Primaria, 5, 629-641.

Elliott, M., Browne, K. \& Kilcoyne, J. (1995). Child Sexual Abuse Prevention: What Offenders Tell Us. Child Abuse and Neglect, 19, 579-594.

Fernández, J., Ruiz A., Comas, Ll., Petibó, M., Ibáñez, M. \& Bassets, J. (2001). Abuso sexual. Experiencia en una Unidad Funcional de abusos a menores. Anales Españoles de Pediatría, 54(3).

Finkelhor, D. \& Brown, A. (1985). The Traumatic Impact of Child Abuse: A Conceptualization. American Journal of Orthopsychiatry, 55(4), 530-541.

Finkelhor, D. (1993). Epidemiological Factors in the Clinical Identification of Child Sexual Abuse. Child Abuse and Neglect, 17, 67-70.

Finkelhor, D. (1994). The International Epidemiology of Child Sexual Abuse. Child Abuse and Neglect, 18(5), 409-417. 
Finkelhor, D. (2005). Abuso Sexual al Menor. México: Ed. Paz México

Fundación León Bloy (2008). Modelo para la alerta temprana de vulneración de derechos en el espacio local 2007-2008. Informe Final. SENAME: Santiago de Chile.

Gladstone, G., Parker, G., Mitchell, P., Malhi, G., Wilhelm, K. \& Austin, M. (2004). Implications of Childhood Trauma for Depressed Women: An Analysis of Pathways. American Journal of Psychiatry, 161, 1417-1425.

González, E., Martínez, V. Leyton, C. \& Bardi, A. (2004). Características de los abusadores sexuales. Revista Sogia, 11(1), 6-14.

Huerta, S., Maric, V. \& Navarro, C. (2002). Factores que intervienen en el impacto del abuso incestuoso sobre la víctima. Terapia Psicológica, 20(2), 38-46.

Intebi, I. (1998). Abuso sexual infantil en las mejores familias. Argentina: Ediciones Granica SA.

López, F., Fuertes, A., Gómez, J. Carpintero, E., Hernández, A. \& Martín, M. J. (1994). Abusos sexuales a menores. Lo que recuerdan de mayores. Madrid: Ministerio de Asuntos Sociales.

Maida, A., Molina, M., Basualto, C. et al (2005). La experiencia de abuso en las madres: ¿Es un predictor de abuso sexual de sus hijos? Revista Chilena de Pediatría, 76(1), 41 - 47.

Navarro, C., Carrasco, E. \& Aliste, M. (2003). Diagnóstico psicosocial de menores víctimas de agresiones sexuales: Un estudio descriptivo. Anales V Congreso Iberoamericano de Psicología Jurídica (pp. 89-108). Santiago, Chile.

Onostre, R. (2000). Abuso sexual en niñas y niños: Consideraciones clínicas. Revista Chilena de Pediatría, 71(4), 368-375.

Páez, L. \& Hernández, H. (2004). Comportamiento del delito sexual en Colombia. Forenses. Instituto Nacional de Medicina Legal y Ciencias Forenses, Bogotá, Colombia.

Pereda, N., Guilera, G., Forns, M. \& Gómez-Benito, J. (2009). The International Epidemiology of Child Sexual Abuse: A Continuation of Finkelhor (1994). Child Abuse \& Neglect, 33, 331-342.

Pereda, N., Polo, P. Grau, N., Navales, N. \& Martínez, M., (2007). Víctimas de abuso sexual en la infancia. Estudio descriptivo. Revista de Estudios de la Violencia, 1.

Poblete, A. \& Varas, Y. (1994). Abuso sexual en la infancia y psicopatología en la mujer. Anales del Primer Congreso Nacional de Mujer y Salud Mental (pp. 204-208).

Pool, A. (2006). Análisis desde el Modelo Traumatogénico de los indicadores gráficos asociados a agresiones sexuales infantiles en la prueba persona bajo la lluvia. Psykhé, 15(1), 45-55.

Redondo, C. \& Ortiz, M., (2005). El abuso sexual infantil. Boletín de la Sociedad de Pediatría de Asturias, Cantabria, Castilla y León, 45, 3-16. [Versión electrónica].

Roesler, T. \& Weisssmann-Wind, T. (1994). Telling the Secret: Adult Women describe their Disclosures of Incest. Journal of Interpersonal Violence, 9, 327-338.

Sánchez, E. \& Martín, I. (2007). Características de una Muestra de Niños con sospecha de Abuso Sexual en un Dispositivo Especializado Andaluz. Intervención Psicosocial, 16(3), 339-359.

Santana, A. \& Farkas, Ch. (2007). Estrategias de Autocuidado en Equipos Profesionales que Trabajan en Maltrato Infantil. Psykhé, 16(1), 77-89.

Terry, K. \& Tallon, J. (2004). Child Sexual Abuse: A Review of the Literature. Washington, D.C.: EEUU.

Trepper, T., Niedner, D., Mika, L. \& Barrett, M. (1994). Family Characteristics of Intact Sexually Abusing Families: an exploratory study. Journal of Child Sexual Abuse, 5, 1-18. 
Trepper, T. \& Barret, M. (1989). Systemic Treatment of incest. Brunner/Mazel, inc. Publishers, New York: EE.UU.

Trocmé, N., MacLaurin, B., Fallon, B., Daciuk, J., Billingsley, D., \& Tourigny, M. (2001). Canadian incidence study of reported child abuse and neglect: Final Report. Canadá.

Urrego, Z. (2007). Las invisibles: una lectura desde la salud pública sobre la violencia sexual contra niñas y mujeres Colombianas en la actualidad. Revista Colombiana de Obstetricia Ginecológica, $58(1), 38-44$. 
